\title{
Dinámica de la regeneración de Austrocedrus chilensis y Nothofagus dombeyi en bosques en decaimiento
}

\author{
Regeneration dynamics of Austrocedrus chilensis and Nothofagus dombeyi \\ in declining forests
}

\author{
Sofía Losada Palenzuela a , Mariano Martín Amoroso ${ }^{\text {bc*, }}$, Stella Marys Bogino ${ }^{\text {d }}$ \\ a Universidad de Valladolid, Escuela Técnica Superior de Ingeniarías Agrarias, España. \\ * Autor de correspondencia: ${ }^{b}$ Universidad Nacional de Río Negro, Instituto de Investigaciones en Recursos Naturales, \\ Agroecología y Desarrollo Rural, Sede Andina, Onelli 3076, El Bolsón, CP 8430, Río Negro, Argentina, mamoroso@unrn.edu.ar

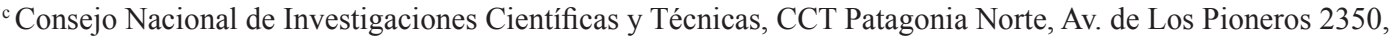 \\ S.C. de Bariloche, CP 8400, Río Negro, Argentina, tel.: +54 2944455455. \\ ${ }^{d}$ Universidad Nacional de San Luis, Departamento de Ciencias Agropecuarias, Avenida 25 de Mayo 384, \\ 5730 Villa Mercedes, San Luis, Argentina.
}

\begin{abstract}
SUMMARY
The Austrocedrus chilensis forests in Argentina experience a process of decline and mortality with important impacts on the structure and population dynamics. Although its study has received significant attention over time, knowledge related to the growth of the regeneration and its response to the gradual changes in the canopy over time driven by mortality is incipient. The objective of this study was to investigate, through dendrochronological methods, the population dynamics and the radial growth of the regeneration of individuals of Nothofagus dombeyi and A. chilensis in declining forests in the northwest of Río Negro Province, Argentina. Overstory mortality and canopy changes significantly impacted the understory structure through the establishment and growth patterns of regeneration. The average radial growth, the average age and the average diameter of the individuals of $A$. chilensis and $N$. dombeyi differed significantly. There is a positive response in the average growth of both species when the basal area loss by mortality exceeds $20 \%$, probably due to the higher number of changes in the instantaneous rates of growth of individuals. Based on our results, we can predict a transition from the current pure and single-cohort forests of A. chilensis towards irregular forests of mixed composition.
\end{abstract}

Key words: Patagonia, mortality, radial growth, dendrochronology.

\section{RESUMEN}

Los bosques de Austrocedrus chilensis en Argentina experimentan un proceso de decaimiento y mortalidad con marcados impactos en la estructura y dinámica poblacional. Si bien su estudio ha recibido significativa atención en el tiempo, el conocimiento relacionado al crecimiento de la regeneración y su respuesta en relación a los paulatinos cambios del dosel en el tiempo dados por la mortalidad es incipiente. El objetivo de este estudio fue investigar, a través de métodos dendrocronológicos, la dinámica poblacional y el crecimiento radial de la regeneración de individuos de Nothofagus dombeyi y A. chilensis en bosques en decaimiento en el noroeste de la provincia de Rio Negro, Argentina. La mortalidad y los cambios del dosel condicionan de manera significativa tanto la estructura del sotobosque a través del establecimiento, como los patrones de crecimiento de la regeneración. El crecimiento radial promedio, la edad promedio y el diámetro promedio de los individuos de A. chilensis y $N$. dombeyi analizados difirieron significativamente. Hubo respuesta positiva del crecimiento medio de ambas especies cuando la pérdida de área basal por mortalidad superó el $20 \%$, dado probablemente por el mayor número de cambios en las tasas instantáneas de crecimiento de la regeneración. Sobre la base de estos resultados se puede predecir una transición desde los bosques puros y coetáneos de A. chilensis hacia formaciones disetáneas e irregulares de composición mixta.

Palabras clave: Patagonia, mortalidad, crecimiento radial, dendrocronología.

\section{INTRODUCCIÓN}

Los bosques de Austrocedrus chilensis (D. Don) CFP. Sern. et Bizzarri (ciprés de la cordillera) en Argentina vienen experimentando desde hace varias décadas un proceso de decaimiento y mortalidad conocido localmente como "mal del ciprés" (Havrylenko et al. 1989, Baccalá et al. 1998, Amoroso y Larson 2010). Este proceso representa, junto con el fuego y la herbivoría, uno de los disturbios más importantes en la dinámica poblacional de los bosques mésicos de $A$. chilensis en la región patagónica (Amoroso et al. 2015). 
A nivel de rodal, este proceso se manifiesta como parches de árboles de diferentes tamaños con distintos niveles de defoliación y amarillamiento de la copa y árboles muertos rodeados de individuos asintomáticos, aunque también es posible encontrar un patrón más desagregado. La progresiva defoliación y posterior muerte de los individuos genera importantes cambios en el dosel y la estructura de los bosques, observándose así árboles muertos en pie y caídos que van generando claros de tamaños variables en el dosel y cambios en el ambiente lumínico del sotobosque. Si bien diferentes trabajos han estudiado la mortalidad en estos bosques (Havrylenko et al. 1989, Rosso et al. 1994, Baccalá et al. 1998), son escasos los estudios que han proporcionado fechas precisas de la muerte de los árboles y abordado los impactos derivados sobre la estructura y la dinámica de estos bosques (Relva et al. 2009, Amoroso y Larson, 2010ab). Respecto a la dinámica poblacional, los estudios reportaron que la mortalidad del dosel favorece la liberación del crecimiento de árboles en el estrato superior y cambios en la composición de las especies y reclutamiento de individuos en el sotobosque a lo largo del tiempo (Relva et al. 2009, Amoroso y Larson 2010b, Amoroso et al. 2012). A su vez, el patrón de mortalidad y las fluctuaciones climáticas (sequías) pueden tener un efecto en la composición del sotobosque favoreciendo el establecimiento de regeneración pura de A. chilensis o mezclas mixtas de A. chilensis y Nothofagus dombeyi (Mirb.) Oersted (Amoroso et al. 2012, 2015). A pesar de estos recientes avances en el estudio de la regeneración en el estrato inferior de estos bosques en decaimiento, ningún trabajo ha estudiado el crecimiento y la respuesta de la regeneración ya establecida en relación a los paulatinos cambios del dosel en el tiempo.

La dendroecología, o aplicación de técnicas dendrocronológicas al estudio de la ecología y dinámica de los bosques, permite obtener información precisa acerca de la dinámica del rodal, de la estructura de edades y el régimen de los disturbios en tiempos pasados (Fritts y Swetnam 1989), incluyendo los cambios en el crecimiento radial de los árboles en respuesta a los disturbios (Amoroso y Larson 2010). El objetivo de este estudio es investigar, a través de métodos dendroecológicos, la dinámica poblacional y el crecimiento radial de la regeneración de individuos de $N$. dombeyi y A. chilensis en bosques afectados por "mal del ciprés" en el Área Natural Protegida Río Azul - Lago Escondido, Río Negro, Argentina. El objetivo de este trabajo es reconstruir la dinámica del crecimiento de la regeneración de las dos especies a través del tiempo, evaluando los efectos que la muerte de individuos del dosel, al incrementar la radiación en el sotobosque, ejerce sobre el establecimiento y el crecimiento de los individuos bajo diferentes niveles de competencia relativa del sotobosque. Este objetivo se basa en la hipótesis de que es posible reconstruir temporalmente, a través de estudios dendrocronológicos, el impacto de la mortalidad de $A$. chilensis sobre la regeneración de los bosques mixtos de. A. chilensis y N. dombeyi. Además, debido a la existencia de distintos grupos funcionales es posible suponer que la respuesta de la regeneración a este disturbio variará de acuerdo con la especie. La integración de todos estos aspectos permitirá complementar el conocimiento de la dinámica poblacional de estos bosques, generar mayor información de base para predecir su desarrollo, y optimizar el manejo de los sistemas silvícolas mediante estrategias de aprovechamiento maderero.

\section{MÉTODOS}

Área de estudio. Se estudiaron rodales dominados por Austrocedrus chilensis ubicados en la Reserva Forestal Loma del Medio - INTA (Instituto Nacional de Tecnología Agropecuaria) en la ciudad de El Bolsón, Río Negro, Argentina $\left(41^{\circ} 46^{\prime} \mathrm{S} ; 71^{\circ} 33^{\prime} \mathrm{O}\right)$. El área tiene una temperatura media anual de $9,3{ }^{\circ} \mathrm{C}$ y una precipitación anual de $900 \mathrm{~mm}$. Los suelos son profundos, constituidos por cenizas volcánicas, clasificándose como Andisoles. Los bosques se caracterizan por ser estructuras coetáneas pos-incendio y estar dominados por A. chilensis con la presencia variable de especies arbóreas de menor tamaño, como Lomatia hirsuta (Lam.) Diels, Maytenus boaria Mol., Aristotelia chilensis (Mol.) Truco y Schinus patagonicus, las cuales frecuentemente forman parte del sotobosque (Dezzoti y Sancholuz 1991).

Muestreo de campo. Se muestrearon cuatro rodales en el año 2007 (cuadro 1) de acuerdo con los siguientes criterios: a) dosel dominado por $A$. chilensis con más del $60 \%$ de área basal, b) porcentaje de individuos con síntomas del "mal del ciprés" igual o superior al $50 \%$ y c) ausencia de evidencias de herbivoría y de aprovechamiento forestal. En cada uno de estos rodales se establecieron parcelas de $1.000 \mathrm{~m}^{2}$ de forma cuadrada $(31,6 \times 31,6 \mathrm{~m})$ o rectangular $(40 \times 25 \mathrm{~m})$ en función de la topografía local. Para todos los individuos con un diámetro a la altura del pecho (dap) mayor que $5 \mathrm{~cm}$, se determinó la especie, se registró el dap y se extrajeron muestras de barreno a $30 \mathrm{~cm}$ del suelo. En cada una de las parcelas se instalaron nueve subparcelas circulares de $30 \mathrm{~m}^{2}$ (3,09 $\mathrm{m}$ de radio) distribuidas equidistantemente cada $10 \mathrm{~m}$. Dentro de cada subparcela, y para todos los individuos arbóreos con una altura mayor que $50 \mathrm{~cm}$ y un dap menor a $5 \mathrm{~cm}$, se determinó la especie, se registró el diámetro en la base, la altura total, y su estado de competencia relativa a partir de una modificación de la clasificación propuesta por Letourneau et al. (2004). Estos autores clasifican a los individuos en "suprimidos", "intermedios" y "dominantes" de acuerdo con el nivel de competencia entre las distintas formas de vida presentes en el sotobosque en el momento del muestreo. Finalmente, se obtuvo una sección transversal a la altura del cuello de cada individuo dentro de las subparcelas. Del total de los individuos muestreados en las subparcelas, se tomó una submuestra de $A$. chilensis y $N$. dombeyi intentado 
garantizar un mínimo de 20 individuos por rodal y especie. Estas submuestras fueron seleccionadas con la intención de obtener muestras de los individuos de regeneración de mayor edad para garantizar una mayor ventana temporal. En las parcelas EU2 y K1 no fue posible alcanzar el mínimo de 20 individuos por submuestra, contando solo con 18 y 12 individuos, respectivamente (cuadro 2).

Procedimiento de laboratorio. Todas las muestras de barreno y secciones transversales fueron pulidas con lija de granulometría en densidad creciente $(60$ a 600 granos $/ \mathrm{cm}^{2}$ ), obteniéndose superficies lisas y libres de imperfecciones hasta lograr una correcta visualización del arreglo anatómico que determina la delimitación de los anillos de crecimiento. El fechado visual se realizó con ayuda de una lupa binocular marca Olympus, modelo SZ61 $(0,9$ a 4 x) iniciando la datación de los anillos desde la corteza hacia la médula. Una vez fechadas las muestras, se procedió a medir el ancho de los anillos de crecimiento en una tableta UniSlide TA 4020H1-S6 Velmex conectada a un contador digital con una precisión de $0,001 \mathrm{~mm}$. Para verificar la calidad del fechado visual se utilizaron anillos marcadores y el programa COFECHA (Holmes 1983). Para facilitar el fechado de las secciones transversales de la regeneración se confeccionaron primeramente cronologías de ancho de anillo para cada uno de los rodales estudiados a partir de las muestras de barreno de los árboles del dosel. Las series de anillos que presentaron valores bajos de correlación con las cronologías de referencia fueron revisadas visualmente y aquellas que no lograron superar valores de correlación del 0,32 fueron descartadas para minimizar el error en los análisis posteriores. La fecha de mortalidad de los individuos adultos del dosel fue determinada como el año calendario que correspondió al último anillo de crecimiento disponible en la muestra.
Análisis de datos. Utilizando las muestras correctamente fechadas y las mediciones de los anillos de crecimiento, se estimó el año de establecimiento y la edad de los árboles del dosel a la altura de muestreo $(30 \mathrm{~cm})$, y se calcularon el crecimiento radial medio e incremento radial por año. A partir de estos datos se procedió a la construcción de curvas de crecimiento radial medio y crecimiento radial acumulado por edad y por año calendario para cada especie y rodal.

Para el total de los individuos de la regeneración muestreados en cada parcela se cuantificó la proporción de individuos por especie según su estado de competencia relativa al momento del muestreo (suprimidos, intermedios y dominantes), y con el objetivo de comparar el crecimiento por su estado de competencia relativa se procedió a calcular los

Cuadro 2. Número de individuos de Austrocedrus chilensis y Nothofagus dombeyi seleccionados en cada uno de los rodales estudiados. AC, Austrocedrus chilensis; ND, Nothofagus dombeyi.

Number of samples of Austrocedrus chilensis and Nothofagus dombeyi obtained for each of the studied stands. AC, Austrocedrus chilensis; ND, Nothofagus dombeyi.

\begin{tabular}{cccc}
\hline Parcela & Especies & N & Total \\
\hline \multirow{2}{*}{ EU1 } & AC & 20 & 46 \\
& ND & 26 & \\
\hline \multirow{2}{*}{ EU2 } & AC & 18 & 38 \\
& ND & 20 & \\
\hline \multirow{2}{*}{ RQ2 } & AC & 20 & \multirow{2}{*}{42} \\
& ND & 22 & \\
\hline \multirow{2}{*}{ K1 } & AC & 22 & \multirow{2}{*}{34} \\
& ND & 12 & \\
\hline
\end{tabular}

Cuadro 1. Características geográficas y estructurales de cuatro rodales en el área de El Bolsón. Las proporciones totales para todas ellas suman 100 \%. R: rodal; A (m): altitud; P (०): pendiente; ७: azimut. AC, Austrocedrus chilensis; ND, Nothofagus dombeyi; LH, Lomatia hirsuta; OT, otras especies como Aristotelia chilensis y Schinus patagonicus. A, asintomáticos; S, sintomáticos; M, muertos. La composición de cada especie (\%) representa la contribución porcentual de cada especie a la densidad total.

Geographical and structural characteristics of four stands in El Bolsón area. The sum of all proportions add up to $100 \%$. R: stand; A (m): altitude; P (०): slope; ९: azimuth. AC, Austrocedrus chilensis; ND, Nothofagus dombeyi; LH, Lomatia hirsuta; OT, other species such as Aristotelia chilensis and Schinus patagonicus. A, asymptomatic; S, symptomatic; M, dead.

\begin{tabular}{|c|c|c|c|c|c|c|c|c|c|c|c|}
\hline \multirow{2}{*}{$\mathrm{R}$} & \multirow{2}{*}{$\mathrm{A}(\mathrm{m})$} & \multirow{2}{*}{$\mathrm{P}\left({ }^{\circ}\right)$} & \multirow{2}{*}{$\vartheta$} & \multirow{2}{*}{$\begin{array}{c}\text { Densidad } \\
\text { (Árboles/ha) }\end{array}$} & \multicolumn{4}{|c|}{ Composición } & \multicolumn{3}{|c|}{ Estado } \\
\hline & & & & & $\mathrm{AC}(\%)$ & ND (\%) & LH (\%) & OT (\%) & A & $\mathrm{S}$ & M \\
\hline EU1 & 420 & 25 & 130 & 1.490 & 67 & 14 & 18 & 1 & 32 & 28 & 40 \\
\hline EU2 & 398 & 20 & 90 & 1.260 & 69 & 7 & 17 & 7 & 28 & 34 & 38 \\
\hline RQ2 & 486 & $0-5$ & 90 & 1.400 & 54 & 15 & 18 & 13 & 45 & 22 & 33 \\
\hline $\mathrm{K} 1$ & 367 & 15 & 130 & 1.050 & 73 & 11 & 7 & 9 & 42 & 22 & 36 \\
\hline
\end{tabular}


promedios en crecimiento radial medio, crecimiento radial acumulado, diámetro y edad por cada una de las categorías. Posteriormente, se compararon los valores promedios de cada una de las variables. Para aquellas variables que presentaron normalidad y varianza homogénea, según las pruebas de Shapiro-Wilk y Levene, respectivamente, se utilizó el análisis de varianza univariante con la prueba posterior de Scheffé. Para las variables que no superaron las pruebas de estadística paramétrica, se aplicaron las pruebas no paramétricas de Wilcoxon y Mann-Whitney para comparación por pares. Para analizar las diferencias entre los valores medios de crecimiento, edad y diámetro de A. chilensis y $N$. dombeyi, se utilizó la prueba $t$-de Student en los casos que presentaron normalidad y varianza homogénea. Finalmente, se estudió la relación entre el crecimiento radial medio de ancho de anillo en función de la edad y el diámetro mediante regresión lineal. Los análisis estadísticos se realizaron con el software IBM $^{\circledR}$ SPSS ${ }^{\circledR}$ STATISTICS v. 20.0 para Mackintosh (IBM Corp. Released 2011).

Con el objetivo de analizar la respuesta del crecimiento radial de la regeneración en función de las progresivas aperturas de dosel como consecuencia de su mortalidad por el "mal del ciprés", se procedió a comparar las cronologías de anchos de anillo por especie de los árboles del dosel, la evolución de la mortalidad de individuos en el dosel y los cambios abruptos en el crecimiento radial (liberaciones) de la regeneración. Las cronologías de índice de ancho de anillo por especie de la regeneración se construyeron a partir del incremento radial anual medio estandarizado. Este proceso de estandarización, dividiendo cada ancho de anillo por el promedio de ancho de la serie, se utilizó para conservar las tendencias de crecimiento de los árboles individuales a largo plazo e independizar sus tasas de crecimiento absolutas, lo que permite la comparación directa de las tasas relativas de crecimiento de árboles de diferentes tamaños y especies (Amoroso et al. 2012). Para cuantificar la evolución de la mortalidad de individuos en el dosel se reconstruyó el área basal total del dosel (viva y muerta) por hectárea en el tiempo. Para construir la curva de mortalidad se utilizaron los valores del porcentaje de mortalidad del área basal (AB) en el dosel obtenidos de la relación del área basal muerta (tanto de árboles en pie como caídos) sobre el total del área basal (ecuación 1; Amoroso y Larson 2010b).

Porcentaje de mortalidad de $\mathrm{AB}=\frac{\mathrm{ABmuerta}}{\text { ABtotal }} \times 100$

Para definir los criterios en los aumentos abruptos del crecimiento radial (liberaciones) y su posterior cuantificación se utilizaron diferentes vías de análisis. En una primera instancia, se graficaron las series individuales de cada renoval de A. chilensis y N. dombeyi. Esto permitió una exploración visual del grado de liberaciones encontradas en los individuos, así como también el tiempo durante el cual el período de liberación resultaba sostenido.
A pesar de que el método de la media móvil (Nowacki y Abrams 1997) tiende a eliminar cambios en el crecimiento no asociados a disturbios en el dosel, se recomienda realizar la inspección visual de las series para confirmar que las tendencias son sostenidas en el tiempo, que sean coincidentes con la perturbación estudiada y que no sean "falsas liberaciones" ocurridas por tendencias climáticas a corto plazo (Rubino y McCarthy 2004, Amoroso y Larson 2010b). Esta exploración previa, también, permitió definir el número mínimo de años durante el cual las liberaciones deben mantenerse en el tiempo. Posteriormente se realizó la determinación del año de ocurrencia y la cuantificación de las liberaciones, utilizando la técnica propuesta por Nowacki y Abrams (1997) mediante el programa JOLTS del DPL (Holmes 1999). Esta técnica detecta liberaciones de los individuos remanentes, calculando el porcentaje de cambio en el crecimiento (PCC) año a año sobre las series individuales mediante la siguiente ecuación [2]:

$$
\mathrm{PCC}=\frac{\mathrm{M} 2-\mathrm{M} 1}{\mathrm{M} 1} \times 100
$$

Donde, $\mathrm{M} 1$ = promedio de ancho de anillos de los cinco años previos incluyendo el año calculado y M2 = promedio de los cinco años siguientes.

Se calcularon los porcentajes de cambio en el crecimiento en cada serie de anillos de todos los árboles. En este estudio se consideraron las liberaciones donde el porcentaje de cambio en el crecimiento superó el 50 y el $100 \%$ y además se mantuvo al menos por dos años consecutivos. Las liberaciones mayores al $50 \%$ se consideraron moderadas y las superiores al $100 \%$ correspondieron a liberaciones altas (Firm et al. 2009). Finalmente, se graficaron los índices de crecimiento de ancho de anillo de las cronologías de A. chilensis y N. dombeyi donde se superpusieron la curva de mortalidad en porcentaje de área basal del dosel, la proporción de ocurrencia de las liberaciones y el número total de árboles que mostraron liberaciones moderadas $(50 \%)$ y altas $(100 \%)$.

\section{RESULTADOS}

Caracteristicas estructurales del sotobosque. El crecimiento radial, la edad y el diámetro de la regeneración de A. chilensis y $N$. dombeyi difirió significativamente (cuadro 3). El ancho medio de anillo y el diámetro promedio de $N$. dombeyi fue significativamente mayor que A. chilensis en todos los rodales con la excepción de EU2 (diámetro) y RQ2 (ancho de anillo). Contrariamente, A. chilensis presentó una edad promedio significativamente mayor que $N$. dombeyi en todos los rodales $(P<0,0001$; cuadro 3$)$. Los resultados, sin embargo, variaron al considerar la competencia relativa entre los individuos de la misma especie. El ancho de anillo medio y la edad de la regeneración de A. chilensis y $N$. dombeyi no fueron significativamente di- 
ferentes entre las categorías de competencia relativa, con la excepción del rodal EU1 donde los individuos de A. chilensis suprimidos presentaron edad significativamente mayor que los intermedios y estos a su vez que los dominantes $(P<0,01$; cuadro 3$)$. El diámetro, por su parte, fue significativamente diferente para las dos especies en la parcela EU2 $(P<0,01$; cuadro 3$)$ y para los individuos suprimidos de $A$. chilensis en el rodal EU1 ( $P<0,01$; cuadro 3$)$.

El crecimiento radial mostró una baja correlación con la edad de la regeneración para las dos especies en todos los rodales (figura 1), en cambio, con el diámetro de los individuos, fue mayor, lineal y positiva para las mismas situaciones.

Dinámica del crecimiento radial de la regeneración. Las cronologías de ancho de anillo arrojaron valores mayores para $N$. dombeyi aunque la extensión de estas fue siempre mayor para A. chilensis (figura 2). En general, A. chilensis tuvo una tendencia de crecimiento lineal mostrando un leve incremento en la última década en algunos rodales (RQ2 y K1), mientras que el crecimiento de $N$. dombeyi fue exponencial (figura 2). Por su parte, las cronologías de crecimiento medio en función de la edad biológica arrojaron que $N$. dombeyi exhibió un mayor crecimiento que $A$. chilensis para todas las edades (figura 2). Vale remarcar que, a pesar de las altas tasas de crecimiento inicial observadas en $N$. dombeyi, el crecimiento en los últimos 10 años ha disminuido considerablemente en todos los rodales analizados.

El crecimiento radial acumulado por especie arrojó patrones similares a los descritos anteriormente (figura 3). Austrocedrus chilensis exhibió, en general, un crecimiento con tendencia lineal y baja variabilidad durante los primeros 40 años, mientras que el crecimiento acumulado de $N$. dombeyi fue del tipo exponencial y mayor al de $A$. chilensis comparado a la misma edad.

Establecimiento y patrones de crecimiento radial. Los cambios en el crecimiento radial de la regeneración fue diferente para las dos especies y con marcadas diferencias en la duración promedio y máxima, dependiendo de la magnitud de las liberaciones ( $\mathrm{PCC}>50 \%$ y $\mathrm{PCC}>$ $100 \%$; cuadro 4). Para ambas especies, las liberaciones moderadas ( $\mathrm{PCC}>50 \%$ ) fueron más frecuentes que las altas (PCC $>100 \%$ ), y los individuos de A. chilensis presentaron mayor número. Cabe señalar que, en los rodales donde las liberaciones moderadas en individuos de A. chilensis alcanzaron su mayor número, las liberaciones de $N$. dombeyi resultaron en los valores más bajos. En relación a la competencia relativa de la regeneración, los individuos dominantes alcanzaron, en general, los mayores porcentajes de liberaciones en ambas especies

Cuadro 3. Ancho de anillo, edad y diámetro promedio por categoría de competencia (D: dominante, I: intermedio, S: suprimido) y totales (T) para la regeneración de Austrocedrus chilensis (AC) y Nothofagus dombeyi (ND) en los sitios de muestreo. Letras diferentes indican diferencias significativas, y fuente normal y cursiva en los superíndices indican agrupación en el análisis estadístico. Grado de significancia: $P<0,01 * ; P<0,001 * * ; P<0,0001 * * *$.

Average ring width, age and average by competition category (D: dominant, I: intermediate, S: suppressed) and totals (T) for regeneration of Austrocedrus chilensis (AC) and Nothofagus dombeyi (ND) for all sampling sites. Different letters indicate significant differences, and normal and italic font in the superscripts indicate grouping in the statistical analysis. Degree of significance: $* P<0.01 ; * * P<0.001 ; * * * P<0.0001$.

\begin{tabular}{|c|c|c|c|c|c|c|c|c|c|}
\hline \multirow{2}{*}{\multicolumn{2}{|c|}{ Parámetro }} & \multicolumn{2}{|c|}{ EU1 } & \multicolumn{2}{|c|}{ EU2 } & \multicolumn{2}{|c|}{ RQ2 } & \multicolumn{2}{|c|}{ K1 } \\
\hline & & \multirow{2}{*}{$\begin{array}{c}A C \\
0,24^{\mathrm{ns}}\end{array}$} & \multirow{2}{*}{$\begin{array}{c}D \\
0,67^{\mathrm{ns}}\end{array}$} & \multirow{2}{*}{$\begin{array}{c}A C \\
0,30^{\mathrm{ns}}\end{array}$} & \multirow{2}{*}{$\begin{array}{c}N D \\
0,65^{\mathrm{ns}}\end{array}$} & \multirow{2}{*}{$\begin{array}{c}A C \\
0,30^{\text {ns }}\end{array}$} & \multirow{2}{*}{$\begin{array}{c}N D \\
0,74^{\mathrm{ns}}\end{array}$} & \multirow{2}{*}{$\begin{array}{c}A C \\
0,35^{\text {ns }}\end{array}$} & \multirow{2}{*}{$\begin{array}{l}N D \\
0,51\end{array}$} \\
\hline 용 & D & & & & & & & & \\
\hline 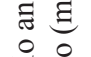 & I & $0,23^{\text {ns }}$ & $0,50^{\mathrm{ns}}$ & $0,28^{\mathrm{ns}}$ & $0,45^{\mathrm{ns}}$ & $0,30^{\mathrm{ns}}$ & $0,64^{\mathrm{ns}}$ & - & - \\
\hline 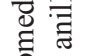 & $\mathrm{S}$ & $0,29^{\text {ns }}$ & $0,31^{\text {ns }}$ & $0,32^{\mathrm{ns}}$ & $0,24^{\text {ns }}$ & $0,37^{\mathrm{ns}}$ & $0,34^{\mathrm{ns}}$ & $0,31^{\text {ns }}$ & - \\
\hline$\stackrel{0}{2}$ & $\mathrm{~T}$ & $0,27^{b^{* *}}$ & $0,64^{a^{* *}}$ & $0,27^{b * *}$ & $0,51^{a^{* *}}$ & $0,30^{n s}$ & $0,61^{n s}$ & $0,34^{b * *}$ & $0,51^{a^{* *}}$ \\
\hline \multirow{4}{*}{ 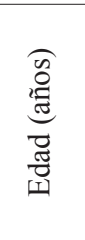 } & D & $25^{c^{*}}$ & $19^{\text {ns }}$ & $48^{\text {ns }}$ & $19^{\text {ns }}$ & $59^{\text {ns }}$ & $21^{\mathrm{ns}}$ & $62^{\text {ns }}$ & 13 \\
\hline & I & $32^{b^{*}}$ & $21^{\text {ns }}$ & $41^{\text {ns }}$ & $20^{\mathrm{ns}}$ & $49^{\text {ns }}$ & $16^{\mathrm{ns}}$ & - & - \\
\hline & $\mathrm{S}$ & $44^{\mathrm{a}^{*}}$ & $23^{\text {ns }}$ & $57^{\mathrm{ns}}$ & $17^{\mathrm{ns}}$ & $59^{\text {ns }}$ & $17^{\mathrm{ns}}$ & $55^{\text {ns }}$ & - \\
\hline & $\mathrm{T}$ & $37^{a^{* * *}}$ & $19^{b^{* * *}}$ & $45^{a^{* * * *}}$ & $19^{b^{* * * *}}$ & $55^{a^{* * * *}}$ & $18^{b^{* * * *}}$ & $60^{a^{* * *}}$ & $13^{b^{* * *}}$ \\
\hline \multirow{4}{*}{ 总 } & D & $11,97^{\mathrm{ab}}$ & $32,56^{\mathrm{ns}}$ & $28,72^{\mathrm{ab}}$ & $29,55^{\text {ab }}$ & $37,29^{\text {ns }}$ & $33,20^{\text {ns }}$ & 44,62 & 15,56 \\
\hline & I & $12,34^{b^{*}}$ & $22,31^{\mathrm{ns}}$ & $19,21^{\mathrm{b}^{*}}$ & $20,20^{a^{*}}$ & $30,56^{\mathrm{ns}}$ & $19,16^{\text {ns }}$ & - & - \\
\hline & $\mathrm{S}$ & $23,99^{a^{*}}$ & $14,21^{\mathrm{ns}}$ & $35,97^{\mathrm{a}^{*}}$ & $8,27^{\mathrm{b}^{*}}$ & $43,39^{\text {ns }}$ & $12,37^{\mathrm{ns}}$ & 36,64 & - \\
\hline & $\mathrm{T}$ & $18,11^{b^{* *}}$ & $30,28^{a^{* *}}$ & $23,84^{n s}$ & $23,55^{n s}$ & $34,57^{a^{*}}$ & $23,56^{b^{*}}$ & $42,81^{a * * *}$ & $15,56^{b^{* * *}}$ \\
\hline
\end{tabular}



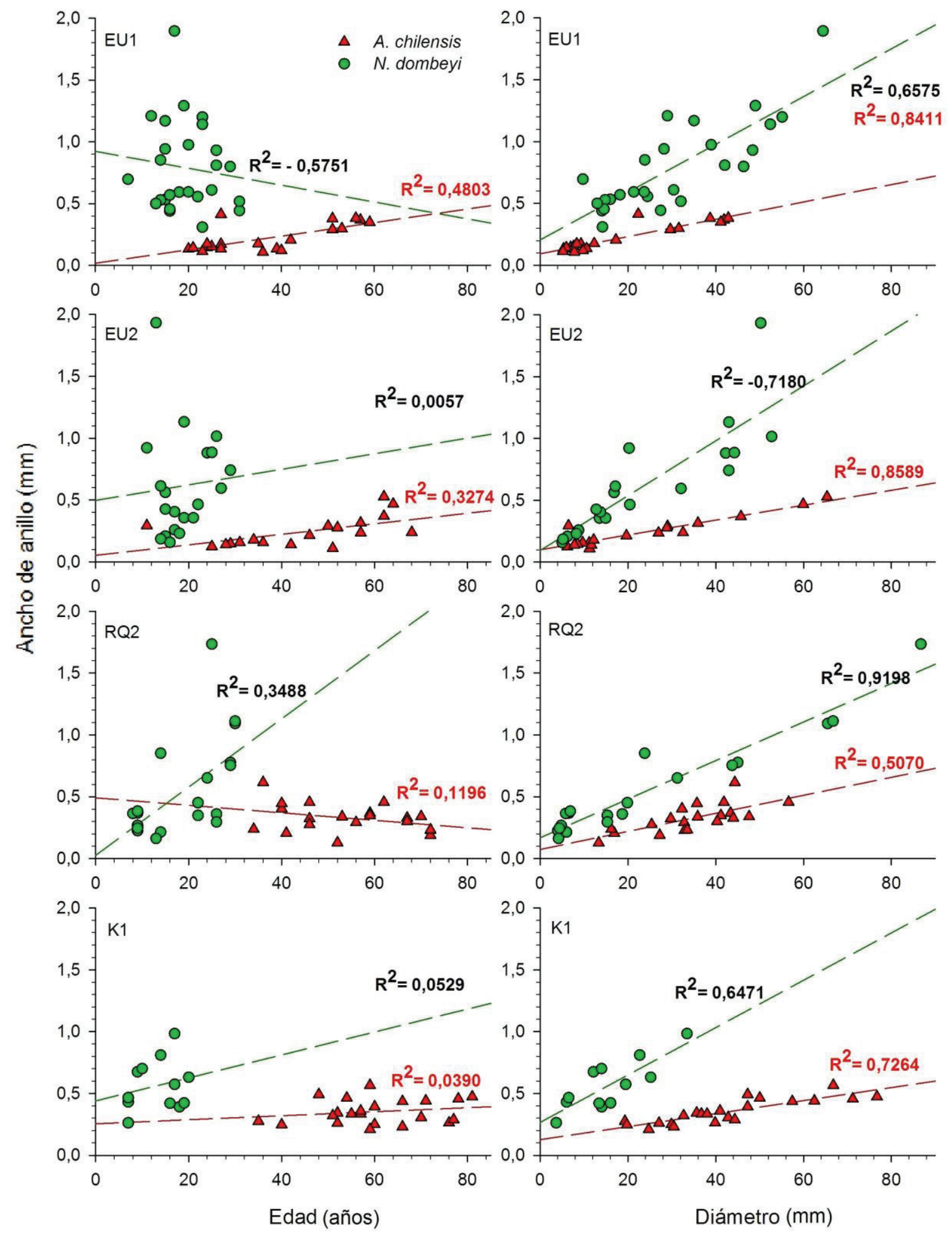

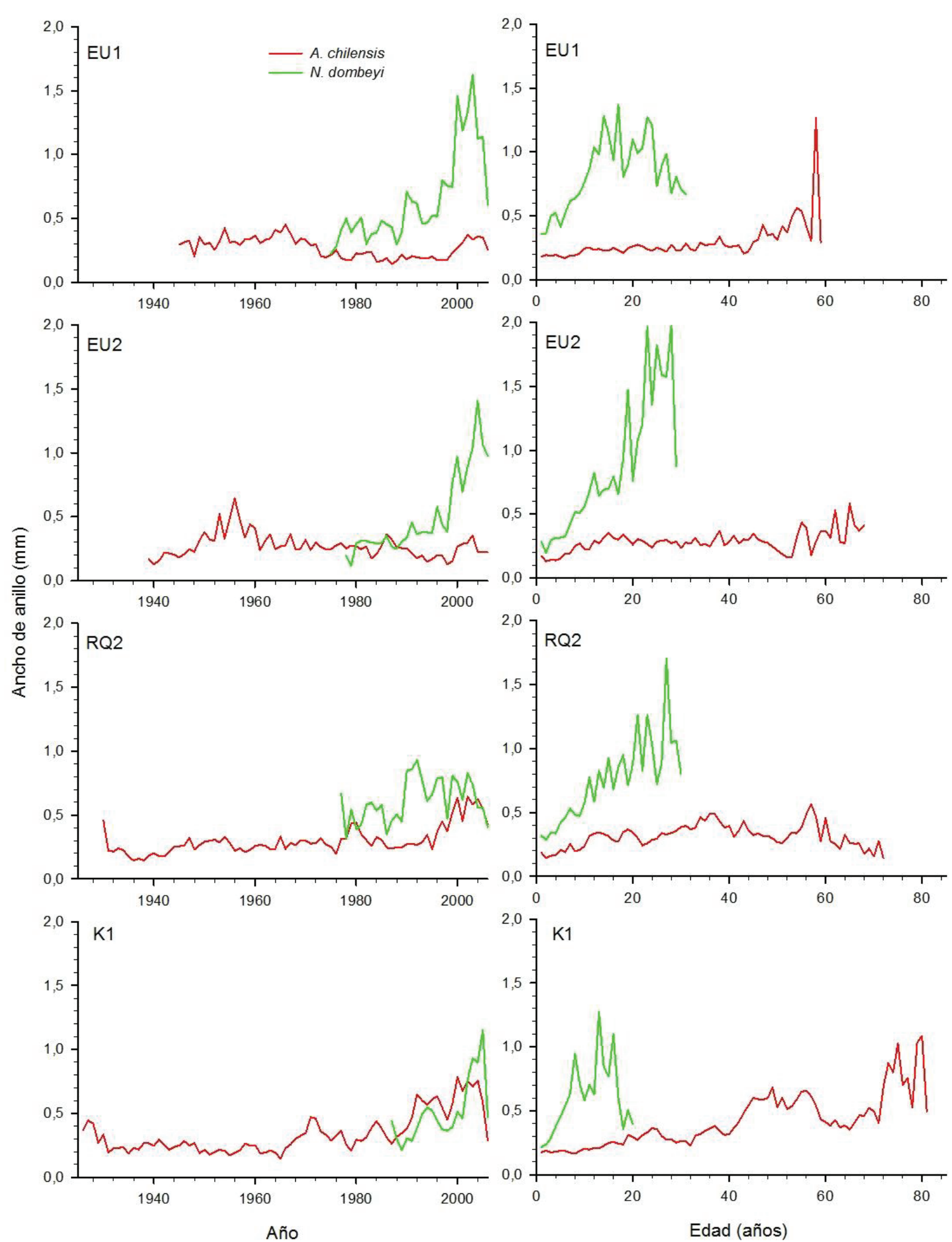

Figura 2. Cronologías de ancho de anillo (mm) de la regeneración de Austrocedrus chilensis (rojo) y Nothofagus dombeyi (verde) en función del año calendario y la edad para cada sitio de muestreo. sampling site.

Ring width chronologies of Austrocedrus chilensis (red) and Nothofagus dombeyi (green) according to the calendar year and age for each 

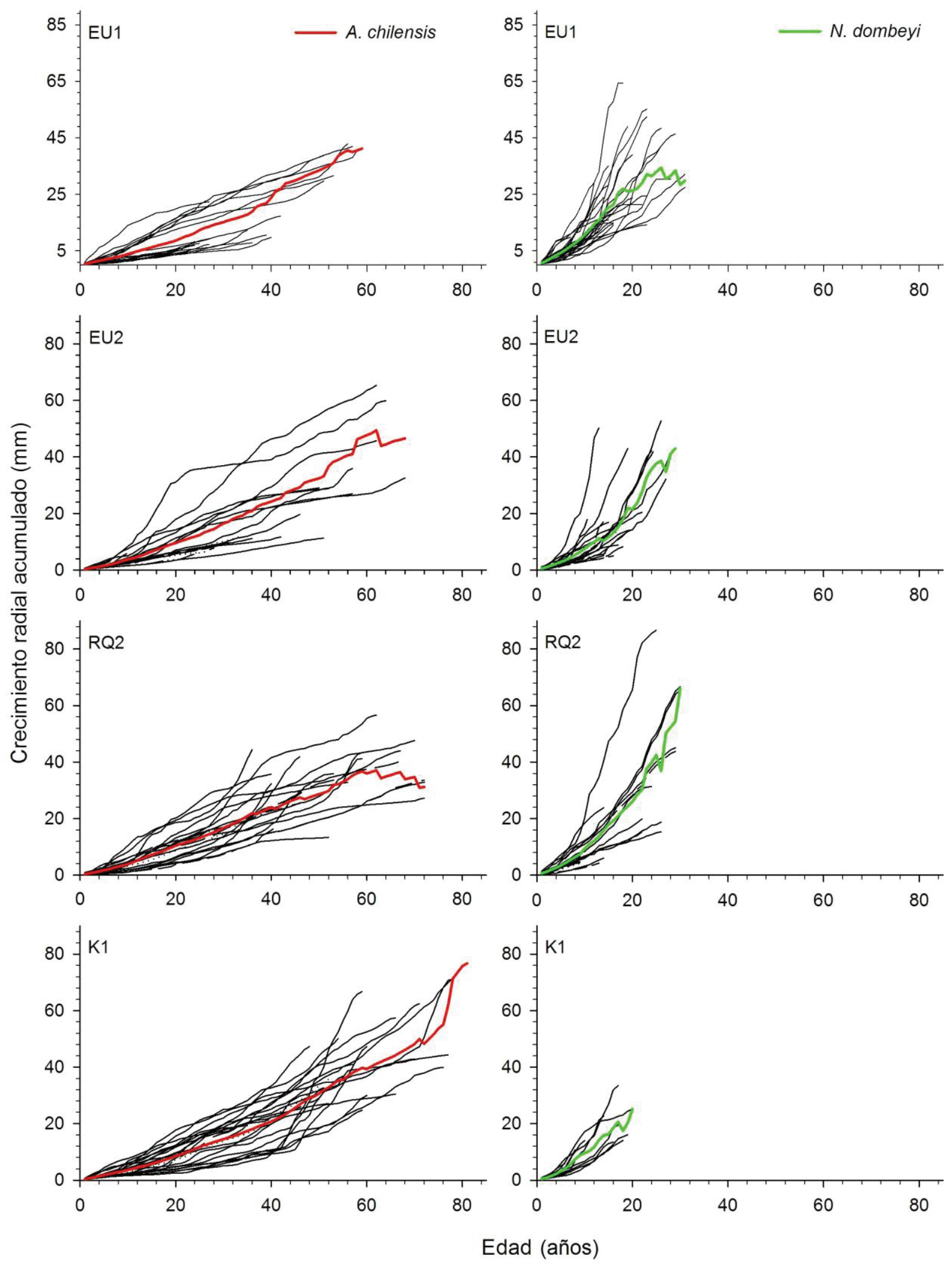

Figura 3. Crecimiento radial acumulado de Austrocedrus chilensis (izquierda) y Nothofagus dombeyi (derecha) por árbol (negro) y promedio (rojo para A. chilensis; verde para $N$. dombeyi) en función de la edad para cada sitio de muestreo.

Cumulative radial growth of Austrocedrus chilensis (left) and Nothofagus dombeyi (right) per tree (black) and average (red for A. chilensis, green for $N$. dombeyi) as a function of age for each sampling site. 
(figura 4). La duración promedio y máxima, en términos generales, no varió considerablemente con la magnitud de las liberaciones para ambas especies (figura 4).

La dinámica del crecimiento radial de la regeneración estuvo condicionada por la mortalidad del dosel aunque con distinta intensidad (figura 4). La ocurrencia y la frecuencia de las liberaciones, en el tiempo, exhibieron un patrón común dado por el inicio y el aumento progresivo del porcentaje de la mortalidad del dosel al igual que por cambios abruptos en esta. Para A. chilensis, particularmen-

Cuadro 4. Proporción, duraciones promedio y máxima de cambios en el crecimiento (liberaciones mayores al 50 y al $100 \%$ ) de la regeneración de Austrocedrus chilensis y Nothofagus dombeyi.

Proportion, average and maximum duration of radial growth changes (releases higher than 50 and $100 \%$ ) of the regeneration of Austrocedrus chilensis (AC) and Nothofagus dombeyi.

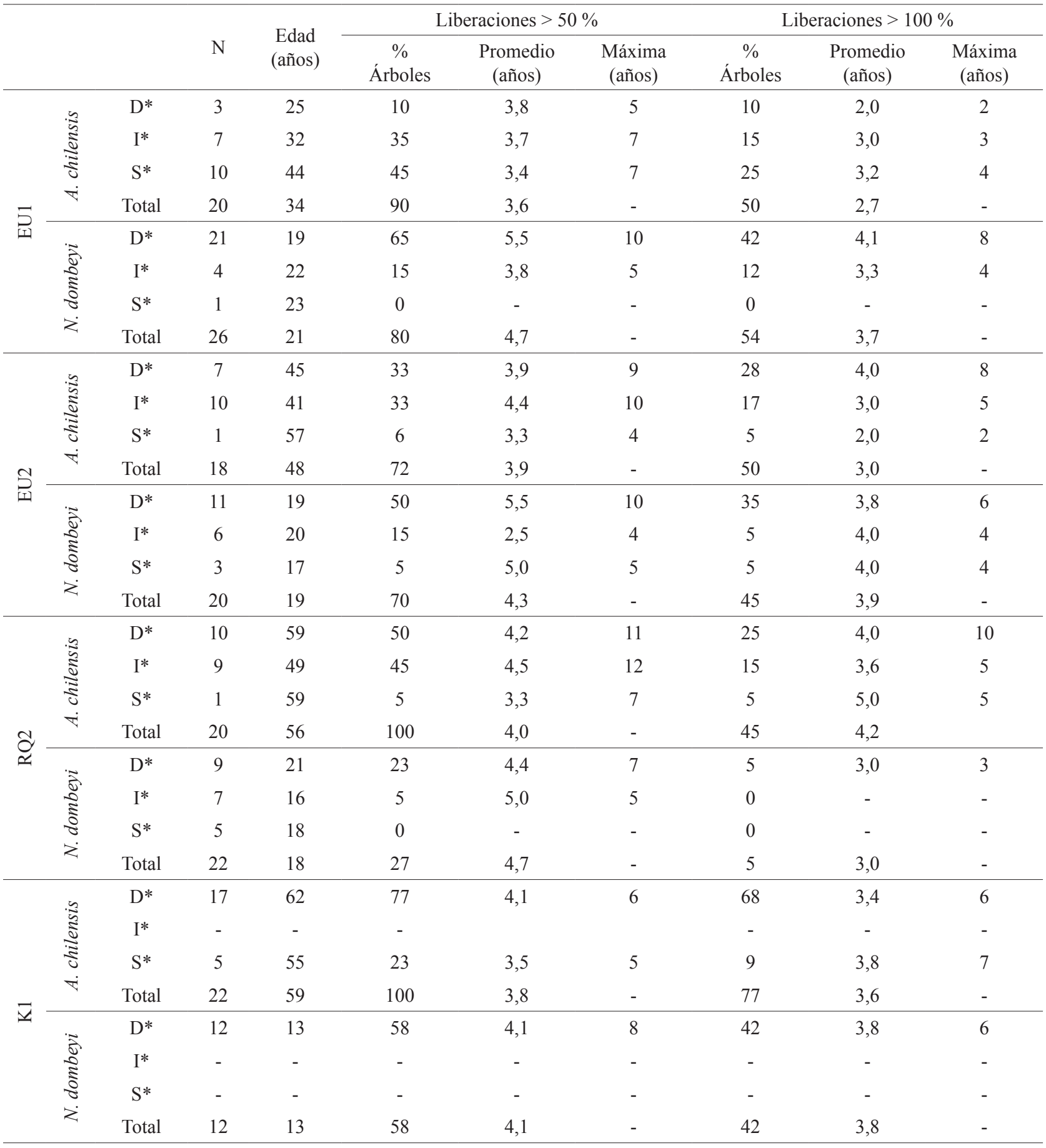



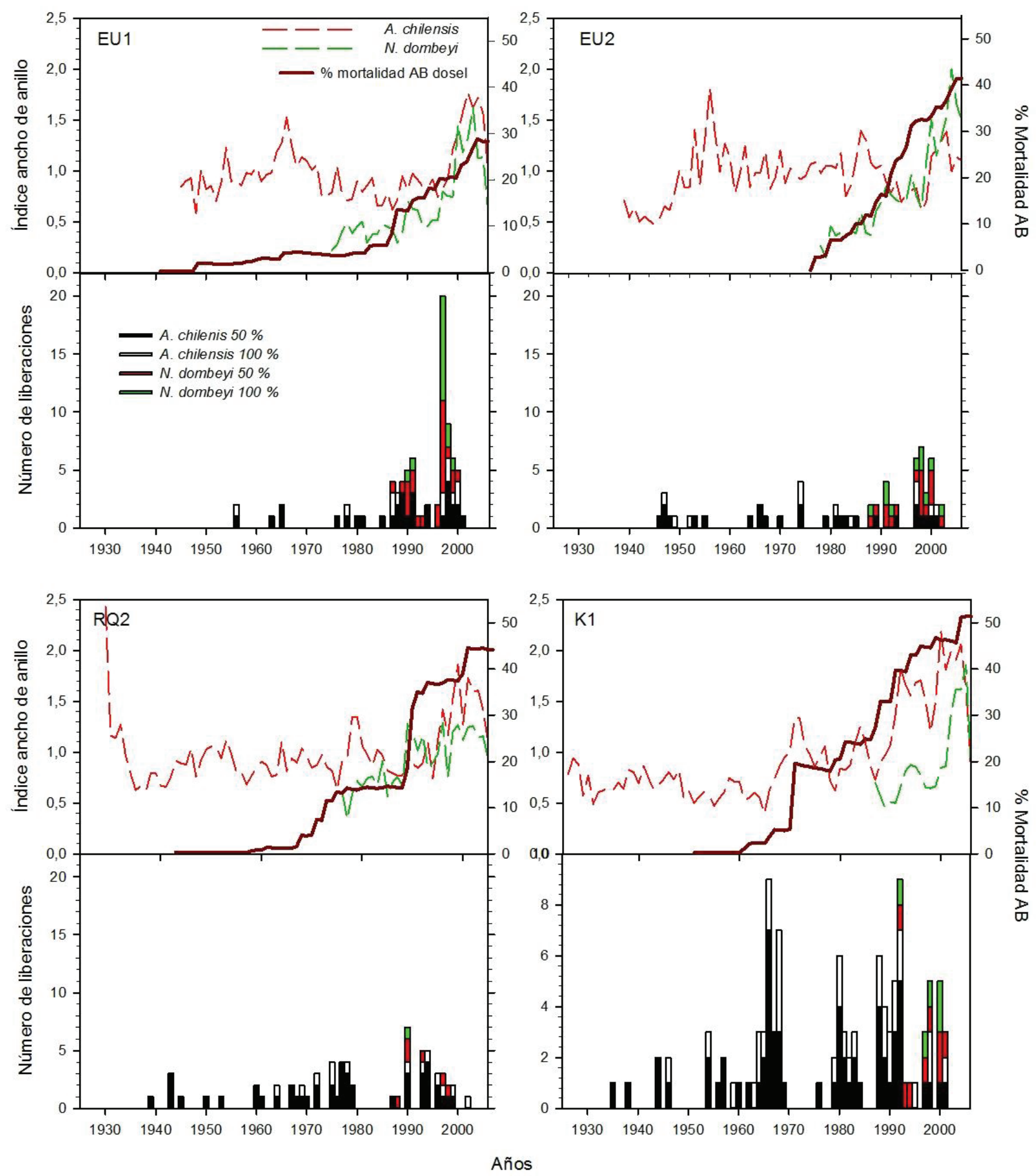

Figura 4. Cronologías de ancho de anillo (líneas discontinuas roja para Austrocedrus chilensis y verde para Nothofagus dombeyi), porcentaje de mortalidad del dosel en área basal (línea continua gruesa marrón), y número de cambios abruptos en el crecimiento (liberaciones) mayores del $50 \%$ y $100 \%$ (color negro y blanco para A. chilensis; color rojo y verde para $N$. dombeyi) en el tiempo para la regeneración de $A$. chilensis y $N$. dombeyi en los sitios de muestreo.

Ring width chronologies (dashed red lines for $A$. chilensis and green for $N$. dombeyi), percentage of basal area canopy mortality (thick brown continuous line), and number of abrupt growth changes (releases) higher than $50 \%$ and $100 \%$ (orange and brown color for $\mathrm{A}$. chilensis, light green and dark green for $N$. dombeyi) at the time for regeneration of Austrocedrus chilensis and Nothofagus dombeyi for all sampling sites. 
te, las liberaciones moderadas se iniciaron sin y con muy bajos porcentajes $(<10 \%)$ de mortalidad (figura 4$)$. Posteriormente, comenzaron a detectarse un mayor número de liberaciones altas coincidiendo con el aumento de la mortalidad. En los individuos de $N$. dombeyi, en general, las liberaciones moderadas y altas comenzaron cuando la mortalidad del dosel alcanzó valores mayores que el $10 \%$. De manera similar, se observó que el aumento de la mortalidad se condijo con un aumento en los índices de ancho de anillo en ambas especies, siendo esta tendencia más marcada para $N$. dombeyi; aún más, la totalidad de la regeneración de esta especie, a diferencia de la de $A$. chilensis, se estableció posteriormente al inicio de la mortalidad en todos los rodales.

\section{DISCUSIÓN}

Los análisis y resultados de este trabajo proveen una interpretación integrada sobre la dinámica de la regeneración de las especies dominantes en bosques de $A$. chilensis en decaimiento. La dendrocronología permitió reconstruir procesos de cambio que ocurrieron en el sistema en los últimos 70 años, permitiendo interpretar el establecimiento de los nuevos individuos, la dinámica temporal del crecimiento de los mismos y su asociación con la pérdida de cobertura del dosel producto del decaimiento y mortalidad de los individuos adultos. La mortalidad y los cambios del dosel asociados en bosques de $A$. chilensis en decaimiento condicionaron, de manera significativa, el establecimiento y los patrones de crecimiento de la regeneración, resultando en respuestas diferenciales por especie. La edad promedio, el diámetro promedio, el crecimiento radial promedio y las curvas de crecimiento de los individuos de $A$. chilensis y $N$. dombeyi analizados difirieron significativamente.

En general, la regeneración de $A$. chilensis se produjo, en promedio, previo al establecimiento de aquella de $N$. dombeyi Las diferencias significativas tanto en cuanto a los períodos de instalación, como en relación a la dinámica de crecimiento de ambas especies, concuerdan con lo reportado previamente para ambas especies bajo estas condiciones (La Manna et al. 2008, Relva et al. 2009, Amoroso y Larson 2010ab, Amoroso et al. 2012). Esta respuesta diferencial puede explicarse como una respuesta específica, dados los diferentes requerimientos lumínicos de las especies a las condiciones ambientales variables acentuadas por los recurrentes eventos de moralidad de ciprés. Mientras que $A$. chilensis puede regenerar bajo dosel cerrado $\mathrm{y}$ en claros de pequeño tamaño por su mayor tolerancia a la sombra, $N$. dombeyi necesita de claros de mayor tamaño (Veblen et al. 1995, Dezzotti 1996, Gobbi y Schlichter 1998, Amoroso et al. 2012).

Si bien el establecimiento de nuevos individuos bajo estas condiciones ya ha sido objeto de estudios previos, el efecto de los cambios estructurales en el dosel, dados por el aumento progresivo de la mortalidad, sobre el crecimiento de la regeneración no había sido aún abordado. Los resul- tados dan cuenta que la apertura del dosel en las últimas 4-5 décadas determinó fuertes cambios en el porcentaje de cambios en el crecimiento radial de los individuos de A. chilensis y $N$. dombeyi establecidos tras la mortalidad; aun cuando el establecimiento de $N$. dombeyi fue más tardío, los individuos presentaron mayor diámetro promedio como resultado de las mayores tasas de crecimiento radial documentadas. Existe una fuerte relación entre el crecimiento medio de ambas especies y los porcentajes de pérdida de área basal en el dosel. En aquellos rodales donde se registraron las tasas más bajas de mortalidad al momento del muestreo, las diferencias absolutas entre el crecimiento medio de las dos especies fue más acentuado, llegando a existir una diferencia del doble entre una y otra. La mayor diferencia en el crecimiento medio de $N$. dombeyi está posiblemente vinculada a su menor tolerancia a la sombra (Veblen et al. 1995), por lo tanto, aún bajo reducciones en el área basal del dosel de baja magnitud, pero permitiendo un mayor ingreso de radiación, su repuesta es muy significativa y diferente de $A$. chilensis. Superados los niveles del $40 \%$ de mortalidad de los árboles del dosel, la diferencia disminuye lentamente ya que los valores medios de crecimiento de $A$. chilensis comienzan a ser más altos mientras que los de $N$. dombeyi permanecen casi constantes. Esta cambiante respuesta podría explicarse por cambios en la tolerancia a la sombra dados por el desarrollo y edad de la regeneración como, indirectamente, a cambios en factores bióticos y abióticos que afecten el crecimiento (Valladares y Niinemets 2008). Aun así, los valores máximos en diámetro los alcanza siempre $N$. dombeyi que a su vez presenta una edad promedio menor. No obstante, los patrones temporales señalan un aumento evidente del crecimiento medio de ambas especies en el momento que se supera el $20 \%$ en pérdida de área basal. Este tipo de respuesta al aumento del tamaño de claros y reducción en la cobertura del dosel ha sido reportada en diferentes ecosistemas boscosos (Brokaw 1987, Coates 2002).

El porcentaje de cambio en el crecimiento radial de la regeneración, por su parte, fue diferente para las dos especies y con marcadas diferencias en la duración promedio y máxima dependiendo de la magnitud de las liberaciones. Para ambas especies, las liberaciones moderadas (PCC $>$ $50 \%$ ) fueron más frecuentes que las altas ( $\mathrm{PCC}>100 \%$ ). El $90 \%$ de A. chilensis y el $60 \%$ de $N$. dombeyi mostraron liberaciones moderadas. Por su parte, solo el $56 \%$ de $A$. chilensis y el $36 \%$ de $N$. dombeyi reflejaron liberaciones altas. Sin duda, la proporción de individuos que respondieron a los cambios en el dosel y la magnitud de la respuesta difirió significativamente de la respuesta habitual de los individuos dominantes en el dosel, donde por lo general esta es menor en número, intensidad y magnitud (Amoroso y Larson 2010b). Al igual con el crecimiento radial medio, el mayor número de liberaciones (incrementos abruptos en el crecimiento radial) en el sotobosque ocurrió cuando se supera el $20 \%$ en pérdida de área basal, reafirmando que los aumentos abruptos en el crecimiento radial de 
la regeneración se ven influenciados por aumentos de la mortalidad una vez que el estrato superior ya presenta un avanzado grado de apertura. Los patrones temporales de ocurrencia, difirieron para cada una de las especies. Mientras que las respuestas de $N$. dombeyi ocurrieron cuando el dosel presentó un alto grado de apertura lo cual está íntimamente ligado a su condición de especie heliófila, las liberaciones de $A$. chilensis pueden ocurrir aún antes del inicio de la mortalidad, posiblemente, como consecuencia de la moderada entrada de luz al sotobosque resultantes de la creciente defoliación de los árboles en decaimiento y que su punto de saturación de luz está por debajo que el $N$. dombeyi; este comportamiento, en el crecimiento de las especies, ya ha sido reportado condiciones similares para árboles del dosel superior (Amoroso y Larson 2010b).

Un resultado de gran importancia por su directa aplicación al manejo silvícola fue la existencia de una relación directa entre el diámetro y el crecimiento promedio del ancho de anillo de la regeneración de ambas especies; los individuos de mayor tamaño presentaron mayores tasas de crecimiento. La contraparte fue la baja relación de la edad con el crecimiento radial de la regeneración en todos los casos. Esto estaría dado, en el caso de $N$. dombeyi, por la alta variabilidad en las tasas promedio de crecimiento para un acotado rango de edades (5-30 años), mientras que para el caso de $A$. chilensis responde a la baja variabilidad en las tasas promedio de crecimiento para un amplio rango de edades (5-80 años). La falta de relación entre la edad y el crecimiento medio también se explica por esta relación con la luz, los individuos de $A$. chilensis son más longevos por su capacidad de instalarse en condiciones de sombra, pero su crecimiento está más restringido, esto explica la falta de relación entre ambas variables. El estado de competencia relativa de los individuos de la regeneración con las distintas especies presentes en el sotobosque, por su parte, no resultó ser un buen predictor del diámetro, el crecimiento y la edad. Esto estaría indicando que la competencia ejercida por el sotobosque sobre la regeneración no influyó sobre el crecimiento de esta. Sin embargo, y como se esperaba, aquellos individuos con menor grado de competencia relativa (dominantes) alcanzaron, en general, los mayores porcentajes de liberaciones altas en ambas especies. La elevada variabilidad en el crecimiento medio anual intraespecífico es determinante cuando se producen liberaciones y esto quedó de manifiesto con mayores tasas de crecimiento radial anual Es decir, el grado de desarrollo de los individuos de la regeneración aparece como un buen predictor del estado de competencia solo en el corto plazo. Este resultado es de mucha utilidad como herramienta de manejo de la futura regeneración, ya que representa un fácil indicador de campo para la selección de los individuos con las mejores posibilidades de crecimiento futuro.

Como ha sido reportado previamente (Amoroso y Larson 2010b, Amoroso et al. 2012), las diferencias absolutas de las tasas de crecimiento medio y el crecimiento acumulado en la regeneración de una especie frente a otra, sumado a la tendencia creciente de la mortalidad del dosel, supone un cambio en la composición del estrato superior de estos bosques en el mediano y largo plazo. Los resultados sugieren que el establecimiento y crecimiento de la regeneración de $A$. chilensis puede verse comprometida con los consiguientes aumentos de la mortalidad del dosel en estos bosques ya que bajo estas condiciones (claros más grandes y menor cobertura) aumentarían las tasas de crecimiento de $N$. dombeyi en detrimento de aquellas de A. chilensis. Si las tendencias observadas continúan, el desarrollo futuro de estos bosques tenderá al desarrollo de masas disetáneas mixtas con estructuras más complejas codominadas por ambas especies, diferentes de los bosques coetáneos actuales dominados por A. chilensis. Una mayor complejidad incluirá la estratificación vertical del dosel. La estratificación se desarrolla naturalmente en rodales de especies mixtas como resultado del crecimiento y la competencia interespecífica y depende de las diferencias en la tolerancia a la sombra, las tasas de crecimiento en altura y las alturas máximas entre las especies (Oliver y Larson 1996). Además, el desarrollo de los rodales estará influenciado por la densidad y la proporción de las especies componentes, atributos que varían entre los bosques afectados por 'mal del ciprés' (Amoroso y Larson 2010b).

\section{CONCLUSIONES}

La reconstrucción de la dinámica del crecimiento de $A$. chilensis y $N$. dombeyi a través de técnicas dendrocronológicas permite documentar la relación existente entre la mortalidad del estrato superior en bosques de $A$. chilensis en decaimiento y las respuestas en el crecimiento de la regeneración. Existe una respuesta positiva del crecimiento medio de ambas especies cuando la pérdida de área basal por mortalidad supera el $20 \%$, dado probablemente por el mayor número de cambios en las tasas instantáneas de crecimiento de la regeneración. Sin embargo, y acorde a la predicción, el crecimiento medio y las liberaciones de ambas especies exhiben diferentes patrones. Las respuestas de $N$. dombeyi a las grandes aperturas del dosel son más inmediatas, aunque retrasadas en su comienzo frente a las liberaciones de $A$. chilensis que responde en mayor número, aunque más lentamente, a las aperturas del dosel. Los individuos de $N$. dombeyi en posiciones dominantes muestran diámetros mayores, y mayores porcentajes en el número de liberaciones y sus máximos sostenidos en el tiempo, respecto del resto de la regeneración. A base del mayor crecimiento medio y acumulado de $N$. dombeyi es de esperar una transición de las actuales masas coetáneas puras de $A$. chilensis hacia masas mixtas irregulares, si las tendencias observadas se mantienen en el tiempo. Esta información ecológica, a su vez, resulta de importancia para el manejo de estos bosques, ya que los cambios en la dinámica documentados y predichos para el desarrollo de masas disetáneas mixtas, que aunque más complejas, plantean nuevas posibilidades de manejo silvícola de estos 
bosques. Por otro lado, las relaciones encontradas representan una buena herramienta de predicción para estimar la estructura y composición del futuro bosque en función de diferentes niveles de intervención silvícola.

\section{REFERENCIAS}

Amoroso MM, BC Larson. 2010a. Can a natural experiment be used as a tool to design partial cutting regimes? The decline of Austrocedrus chilensis forests, an example. Journal of Forest Research 15: 38-45.

Amoroso MM, BC Larson. 2010b. Stand development patterns as a consequence of the mortality in Austrocedrus chilensis forests. Forest Ecology and Management 259: 1981-1992.

Amoroso MM, M L Suarez, LD Daniels. 2012. Nothofagus dombeyi regeneration in declining Austrocedrus chilensis forests: effects of overstory mortality and climatic events. Dendrochronologia 30: 105-112.

Amoroso MM, LD Daniels, R Villalba, P Cherubini. 2015. Does drought incite tree decline and death in Austrocedrus chilensis forests? Journal of Vegetation Science 26: 1171-1183.

Baccalá NB, PH Rosso, M Havrylenko. 1998. Austrocedrus chilensis mortality in the Nahuel Huapi National Park (Argentina). Forest Ecology and Management 109: 261-269.

Brokaw NVL. 1987. Gap-phase regeneration of three pioneer tree species in a tropical forest. Ecology 75: 9-19.

Coates KD. 2002. Tree recruitment in gaps of various size, clearcuts and undisturbed mixed forest of interior British Columbia, Canada. Forest Ecology and Management 155: 387-398.

Dezzotti A. 1996. Austrocedrus chilensis and Nothofagus dombeyi stand development during secondary succession, in northwestern Patagonia, Argentina. Forest Ecology and Management 89: 125-137.

Dezzotti A, L Sancholuz. 1991. Los bosques de Austrocedrus chilensis en Argentina: ubicación, estructura y crecimiento. Bosque 12(1): 43-52.

Firm D, TA Nage, J Diaci. 2009. Disturbance history and dynamics of and old-growth mixed species mountain forest in the Slovenian Alps. Forest Ecology and Management 257: 1893-1901.

Fritts HC, TW Swetnam. 1989. Dendroecology: a tool for evaluating variations in past and present forest environments. Advances in Ecological Research 19: 111-188.

Gobbi M, T Schlichter. 1998. Survival of Austrocedrus chilensis seedlings in relation to microsite conditions and forest thinning. Forest Ecology and Management 111: 137-146.

Havrylenko M, PH Rosso, SB Fontenla. 1989. Austrocedrus chilensis: contribución al estudio de su mortalidad en Argentina. Bosque 10(1): 29-36.

Holmes RL. 1983. Computer-assisted quality control in tree-ring dating and measurement. Tree-Ring Bulletin 43: 69-78.

Holmes RL. 1999. Program JOLTS: Finding growth surges or suppressions in trees. Tucson, Arizona. Laboratory of TreeRing Research, University of Arizona.

IBM Corp. Released. 2011. IBM SPSS Statistics for Windows, Version 20.0. Armonk, NY, USA. IBM Corp.

La Manna L, M Collantes, J Bava, M Rajchenberg. 2008. Natural regeneration in declining Austrocedrus chilensis forests of Patagonia. Ecología Austral 18: 27-41.

Letourneau FJ, E Andenmatten, T Schlichter. 2004. Effect of climatic conditions and tree size on Austrocedrus chilensisshrub interactions in northern Patagonia. Forest Ecology and Management 191: 29-38.

Lorimer CG, LE Frelich. 1989. A methodology for estimating canopy disturbance frequency and intensity in dense temperate forests. Canadian Journal of Forest Research 19: 651-663.

Nowacki GJ, MD Abrams. 1997. Radial-growth averaging criteria for reconstructing disturbance histories from presettlement-origin oaks. Ecological Monographs 67: 225-249.

Oliver CD, BC Larson. 1996. Forest Stand Dynamics. New York, USA. Wiley. $488 \mathrm{p}$.

Relva MA, CL Westerholm, T Kitzberger. 2009. Effects of introduced ungulates on forest understory communities in northern Patagonia are modified by timing and severity of stand mortality. Plant Ecology 201: 11-22.

Rosso PH, NB Baccalaá, M Havrylenko, S Fontenla. 1994. Spatial pattern of Austrucedrus chilensis wilting and the scope of autocorrelation analysis in natural forests. Forest Ecology and Management 67: 273-279.

Rubino DL, BC McCarthy. 2004. Comparative analysis of dendroecological methods used to assess disturbance events. Dendrochronologia 21: 97-115.

Valladares F, U Niinemets. 2008. Shade Tolerance, a Key Plant Feature of Complex Nature and Consequences. Annual Review of Ecology, Evolution, and Systematics 39: 237-257.

Veblen TT, B Burns, T Kitzberger, A Lara, R Villalba. 1995. The ecology of the conifers of Southern South America. In Enright NJ, RS Hill, eds. Ecology of the Southern Conifers. Victoria, Canada. Melbourne Univ. Press. p. 120-155. 
